\title{
Image Enhancement Techniques using Highpass and Lowpass Filters
}

\author{
Aziz Makandar \\ Professor, \\ Department of Computer Science, \\ Karnataka State Women's University, Vijayapur
}

\author{
Bhagirathi Halalli \\ Research Scholar, \\ Department of Computer Science, \\ Karnataka State Women's University, Vijayapur
}

\begin{abstract}
Digital image processing refers to the process of digital images by means of digital computer. The main application area in digital image processing is to enhance the pictorial data for human interpretation. In image acquisition some of the unwanted information is present that will be removed by several preprocessing techniques. Filtering helps to enhance the image by removing noise. The aim of this paper is to demonstrate the lowpass and highpass filtering techniques, however they are the filtering techniques used in Fourier and Wavelet Transformations. In Wavelet Transform these two filters play an important role in reconstructing the original image by using subband coding. Lowpass filter will produce a Gaussian smoothing blur image, in the other hand, high pass filter will increase the contrast between bright and dark pixel to produce a sharpen image.
\end{abstract}

\section{General Terms}

Digital image processing, Image enhancement.

\section{Keywords}

Fast Fourier Transform (FFT), Lowpass Filter, Highpass Filter, Wavelet Transform.

\section{INTRODUCTION}

Interest in digital image processing strategies stems from two principal application areas, improvement of pictorial data for human interpretation and process of image knowledge for storage, transformation and illustration for autonomous machine perception. the target of image improvement is to enhance the standard of image as perceived by human beings through improvement algorithms. Image enhancement is performed each within the spatial domain in addition as in time domain [6].

An image is also outlined as a two-dimensional function $f(x$, $y$ ) where $x$ and $y$ area unit spatial coordinates, and the amplitude of $f$ at any combine of coordinates $(x, y)$ is named the intensity or gray level of the image at the purpose. When $x, y$ and the amplitude values of $f$ are all finite discrete quantities we have a tendency to call them as digital images. The sector of digital image process refers to process digital images by means that of digital computers [1] [2]. However, some digital images are blurred attributable to noise and henceforward images are required to be enhanced for more process. Thus filtering technique is wide accustomed enhancing the images by reducing the noise and more sharpening them. The filters are to reduce digital image improvement technique accustomed sharper the image and to cut back the noise in the image [1-9].

\section{LITERATURE SURVEY}

Randall B [5] High frequency introduced noise in image that decrease image quality and lowpass will smooth the image and reduction noise. Omeed [16] proposed lowpass and highpass filters using java, explained how to implement kernel structure to generate lowpass and highpass filters. Handley [18] showed that the low-pass used for image smoothing and noise reduction. Lowpass effect is to calculate the average of a pixel and all of its eight neighbors. Kenny Hunt [4] proposed that lowpass filtering is convolution that attenuates high frequency of an image while allowing low frequency passing. By the literature review we come to know that lowpass and highpass filters are performing convolution between two array sets which are image elements and filtering mask. By this property lowpass and highpass filters are good to use in image transformation.

\section{FAST FOURIER TRANSFORM}

Fourier Transform is an integral transform of one function into another. Jean Baptiste Joseph Fourier (1768-1830), a French mathematician and physicist introduced Fourier transform. The FFT has played an important role in image processing for many years [1] [2] [6]. The 2D Fourier Transform is a powerful tool and is used to enhance, restore, encode and describe the images. The FFT version is also available and is used in a number of image processing applications to reduce computational cost. The FFT is easy to implement by employing successive doubling technique and hence it finds an important place in image processing applications.

Let $f(x)$ is a continuous function of a real variable $x$. The Fourier Transform of $f(x)$, denoted by $F(u)$ is defined as

$$
F(u)=\int f(x) e^{-j 2 \pi u x} d x \quad \text { where } j=\sqrt{-} 1
$$

Inverse Fourier Transform:

$$
f(x)=\int F(u) e^{-j 2 \pi u x} d u
$$

The two dimensional Fourier transform and its inverse. Fourier transforms in continuous case.

$$
F(u, v)=\iint f(x, y) e^{-j 2 \pi(u x+v y)} d x d y
$$

Inverse Fourier Transforms:

$$
f(x, y)=\iint F(u, v) e^{j 2 \pi(u x+v y)} d u d v
$$

\subsection{Filters}

There are two types of enhancement techniques called spatial domain and frequency domain techniques which are categorized again for smoothing and sharpening the images[19]. We considered the filtering in frequency domain using FFT, use of the terms frequency domain and frequency components is really no different from the terms time domain and time components, which we would use to express the domain and values of $f(x)$ if $x$ where a time variable [1]. 


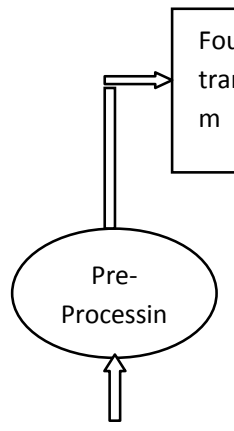

$\mathrm{F}(x, y)$

Input Image

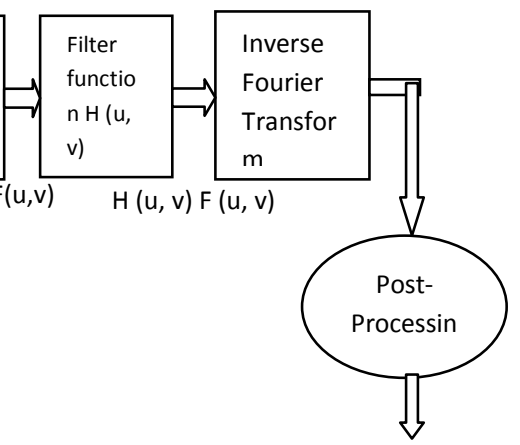

$\mathrm{g}(\mathrm{x}, \mathrm{y})$

Enhanced Image
Fig 1: Frequency Domain Filtering Operation

\subsubsection{Lowpass filter (smoothing)}

A low-pass filter is a filter that passes low-frequency signals and attenuates signals with frequencies higher than the cut-off frequency. The actual amount of attenuation for each frequency varies depending on specific filter design. Smoothing is fundamentally a lowpass operation in the frequency domain [1] [6] [20]. There are several standard forms of lowpass filters are Ideal, Butterworth and Gaussian lowpass filter.

\section{Ideal Lowpass Filters}

The simplest lowpass filter is a filter that "cuts off" all highfrequency components of the Fourier Transform that are at a distance greater than a specified distance $\mathrm{D}$ from the origin of the Transform [17].

The transfer function of an ideal lowpass filter

$$
H(u, v)=\left\{\begin{array}{l}
1 \text { if } D(u, v) \leq D 0 \\
0 \text { if } D(u, v)>D 0
\end{array}\right.
$$

where $D(u, v)$ : the distance from point $(u, v)$ to the center of their frequency.

$$
D(u, v)=\left[\left(u-\frac{M}{2}\right)^{2}+\left(v-\frac{N}{2}\right)^{2}\right]^{\frac{1}{2}}
$$

Butterworth Lowpass Filters: Design frequency-domain filter to remove high-frequency noise with minimal loss of signal components in the specified pass-band with order $n$

$$
H(u, v)=\frac{1}{1+\left[\frac{D(u, v)}{D 0}\right]^{2 n}}
$$

Gaussian Lowpass Filters: The transfer function of a Gaussian lowpass filter is defined as:

$$
H(u, v)=e^{-D^{2}(u, v) / 2 D_{0}^{2}}
$$

\subsubsection{Highpass filters (sharpening)}

A high-pass filter is a filter that passes high frequencies well, but attenuates frequencies lower than the cut-off frequency. Sharpening is fundamentally a highpass operation in the frequency domain.

There are several standard forms of highpass filters such as Ideal, Butterworth and Gaussian highpass filter. All highpass filter $\left(H_{h p}\right)$ is often represented by its relationship to the lowpass filter $\left(H_{l p}\right)$ :

$$
H h p=1-H l p
$$

\section{EXPERIMENTAL RESULTS}

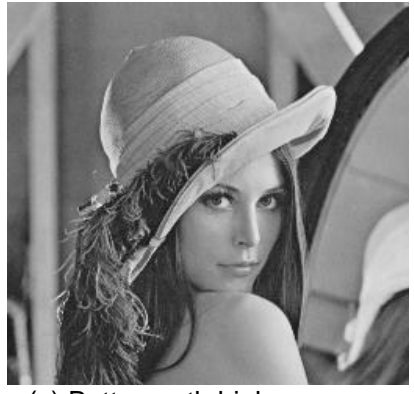

(c) Butterworth highpass

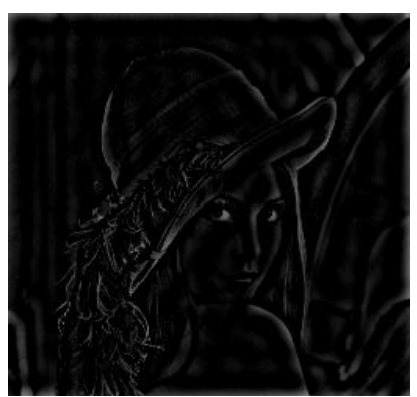

(a) Original Image

(b)Gaussian highpass

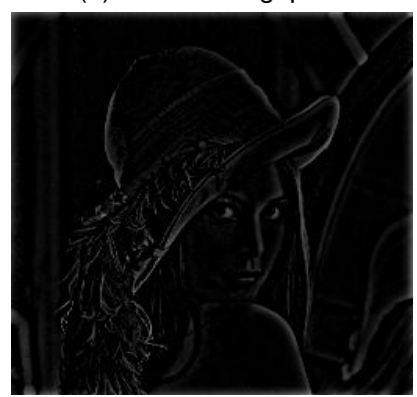

(d)Ideal highpass

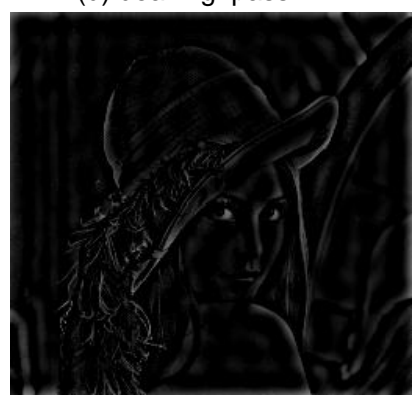

Fig 2: High-pass filters result

In experimental, it shows in figure 4 that RMSE of Gaussian lowpass filter is 15.9687 , Butterworth is 15.9687 and Ideal is 15.9687 and in figure 5 the PSNR of Gaussian is 36.1321, butter worth is 36.1321 and ideal is 36.1321 . By these values we can say that lower RMSE and higher PSNR is of Gaussian lowpass filter, which is good to sharpen the image while transformation.

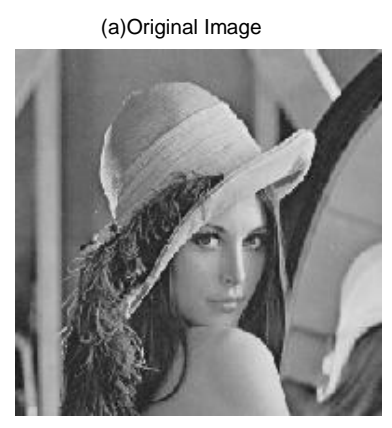

(c) Butterworth Lowpass

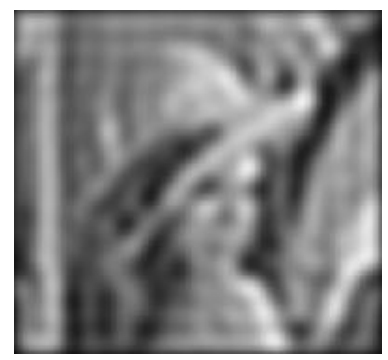

(b)Gaussian Lowpass

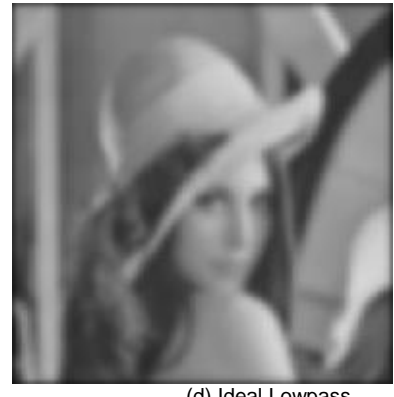

(d) Ideal Lowpass

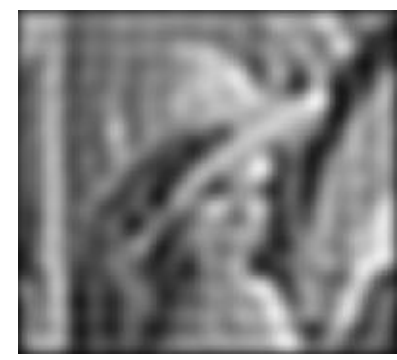

Fig 3: Low-pass filters result
In experimental shown in figure 4. RMSE of Gaussian lowpass filter (fig3 (b)) is 7.7957, Butterworth is 8.5645 and Ideal is 8.6164 and PSNR (figure 5) of Gaussian is 39.2463, butterworth is 38.8378 and ideal is 38.8115 . By these values we can say that lower RMSE and higher PSNR is of Gaussian lowpass filter, which is good to smoothen the image while transformation. 


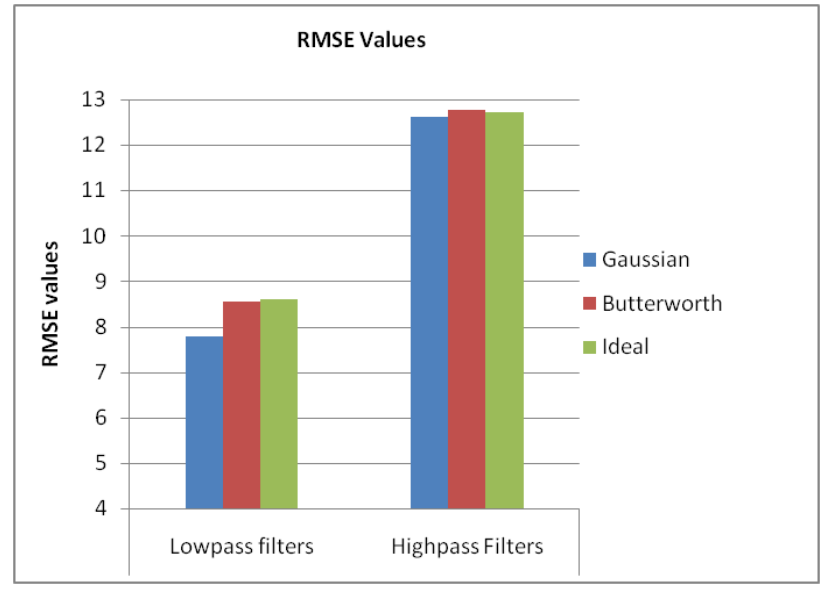

Fig 4. RMSE values of Lowpass filters and Highpass filters

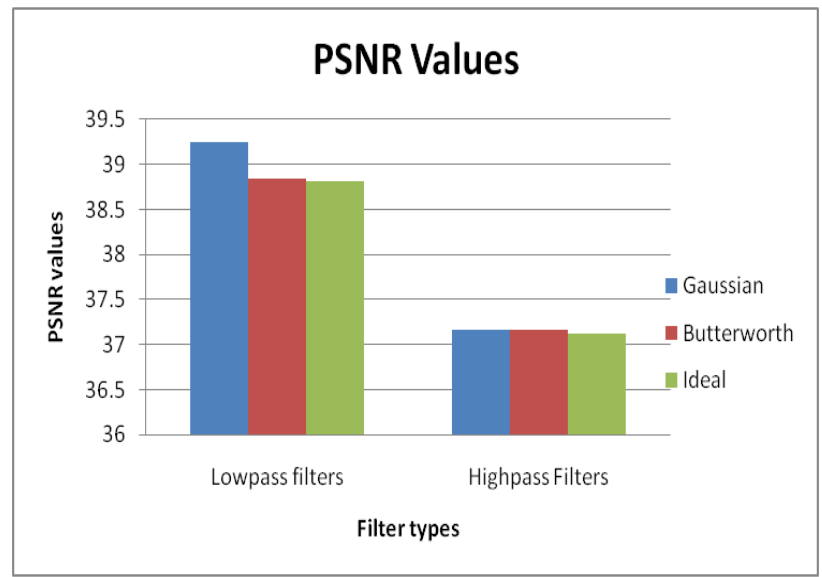

Fig.5: PSNR values of Lowpass and Highpass filters

\section{CONCLUSION}

In this paper Lowpass and Highpass filters are implemented to show the importance of both filters in Fourier and Wavelet Transform. The experimental results show that, highpass filter preserves the edge details and lowpass filter denoises the image by preserving details, therefore highpass and lowpass filters are basic filters used in the image transformations. In both lowpass and highpass filters, Gaussian filter is more suitable for transformation because it has minimum possible group daily and processes in the ideal time domain. It has minimum RMSE and maximum PSNR values which tells about the goodness of it as shown in the result. This method further can be implemented for enhancement of reflectance and illumination effects.

\section{REFERENCES}

[1] R.Gonzalez \& R.Wood, "Digital Image Processing," 3rd ed, Englewood Cliffs, NJ: Prentice Hall, 2007.

[2] BH Brinkmann, A Manduca, RA Robb, "Optimized homomorphic unsharp masking for MR grayscale inhomogeneity correction," Medical Imaging, IEEE Transactions on journal on Medical imaging, Vol. 17. 2011.

[3] J.S. Lee, "Digital image enhancement and noise filtering by use of local statistics," IEEE Transactions on Pattern Analysis and Machine Intelligence, vol. 2, no. 3, pp. 165168.
[4] Randall B. Smith, "Filtering Images," Ph. D thesis 5 January 2012 OMicroImages, http://www.microimages.com/documentation/Tutorials/fi lter.pdf

[5] J.S. Lee, "Digital image smoothing and the sigma filter," Computer Vision, Graphics and Image Processing, vol. 24, pp. 255-269, 1983

[6] Roopashree.S, Sachin Saini, Rohan Ranjan Singh, "Enhancement and Pre-Processing of Images Using Filtering," International Journal of Engineering and Advanced Technology (IJEAT), Volume-1, Issue-5, June 2012

[7] A. Polesel, G. Ramponi and V. J. Mathews, "Image Enhancement via Adaptive Unsharp Masking," IEEE Trans. Image Processing, Vol. 9, No. 3, pp. 505-510, March 2000.

[8] Buades A., Coll B. and Morel J.M Ozaki, Y. Adachi, Y. Iwahori, and N. Ishii, Application of fuzzy theory to writer recognition of Chinese characters, International Journal of Modelling and Simulation, 18(2), pp 112-116, 1998.

[9] Aziz Makandar, Daneshwari Mulimani, Mahantesh Jevoor, "Comparative Study of Different Noise Models and Effective Filtering Techniques," International Journal of Science and Research (IJSR), Volume 3 Issue 8,pp 458- 464, August 2014

[10] J. K. Romberg, M. B. Wakin, and R. G. Baraniuk, "Multiscale geometric image processing," in Proceedings of the SPIE: Visual Communications and Image Processing 2003, pp. 1265-1272, 2003.

[11] R. R. Coifman and D. L. Donoho, "Translation invariant de-noising: Wavelets and statistics," NewYork: Springer-Verlag, 1995.

[12] A. L. Da Cunha, J. Zhou, and M. N. Do, "The Nonsubsampled Contourlet Transform: Theory, Design,and Applications," IEEE Trans. Image Process, vol. 15, pp.3089-3101, 2006.

[13] D. Donoho, I. Johnstone, G. Kerkyacharian, D. Picard, "Wavelet shrinkage: asymptopia?," Journal of the Royal Statistical Society B, vol.57, pp. 301-369, 1995

[14] Philippe Cattin, "Image Restoration: Introduction to Signal and Image Processing," MIAC, University of Basel.

[15] O. Ozsen, "Early Detection of Breast Cancer Using Mathematical Morphology," in Knowledge-Based Intelligent Information and Engineering Systems, pp. 583-590, 2004.

[16] Omeed Kamal Khorsheed, "Produce low-pass and highpass image filter in java," International Journal of Advances in Engineering \& Technology, pp. 712-722, July 2014

[17] Image Processing - Laboratory 9, "Image filtering in the spatial and frequency domains," Technical University of Cluj-Napoca

[18] Nagao M and Matsuyama T. (1997), "Computer Graphics and ImageProcessing,” vol. 9, pp. 394-407. 
[19] Robert Fisher, Simon Perkins, Ashley Walker, Erik Wolfart, 2004, "The Hypermedia Image Processing Reference,'

http://homepages.inf.ed.ac.uk/rbf/HIPR2/unsharp.htm

[20] B.S. Anami, D.G.Savakar, Aziz Makandar, and P.H. Unki (2005), “A Neural Network Model for Classification of Bulk Grain Samples Based on HSI and
Texture," in proceedings of International Conference on Cognition and Recognition, pages 359-368.

[21] Aghagolzadeh S. and Ersory O.K, "Transform image Enhancement," Optical Engineering, vol.31, pp. 614626,1992 .

[22] Herman J. Blinchikoff, Anatol I. Zverev, "Filtering in the Time and Frequency Domains". 